Société d'histoire de la révolution de 1848 et des

révolutions du XIXe siècle

$25 \mid 2002$

Le temps et les historiens

\title{
Culture, croyances et médecine (XIXe-XXe siècle)
}

Mémoire pour le diplôme d'Habilitation à diriger des recherches, sous le tutorat d'Alain Corbin, Université Paris 1-Panthéon-Sorbonne, 2 volumes, $485 \mathrm{f}^{\circ}$, soutenu le 4 juin 2002 devant un jury constitué de Philippe Boutry, Jacqueline Carroy, Alain Corbin, Olivier Faure, JeanYves Mollier (président) et Michelle Perrot.

\section{Nicole Edelman}

\section{(2) OpenEdition} Journals

Édition électronique

URL : http://journals.openedition.org/rh19/453

DOI : $10.4000 /$ rh 19.453

ISSN : $1777-5329$

Éditeur

La Société de 1848

Édition imprimée

Date de publication : 1 décembre 2002

Pagination : 255-262

ISSN : 1265-1354

Référence électronique

Nicole Edelman, «Culture, croyances et médecine (XIXe-XXe siècle) », Revue d'histoire du XIXe siècle [En ligne], 25 | 2002, mis en ligne le 19 juin 2004, consulté le 19 avril 2019. URL : http:// journals.openedition.org/rh19/453 ; DOI : 10.4000/rh19.453

Ce document a été généré automatiquement le 19 avril 2019

Tous droits réservés 


\section{Culture, croyances et médecine (XIXe-XXe siècle)}

Mémoire pour le diplôme d'Habilitation à diriger des recherches, sous le tutorat d'Alain Corbin, Université Paris 1-Panthéon-Sorbonne, 2 volumes, $485 \mathrm{f}^{\circ}$, soutenu le 4 juin 2002 devant un jury constitué de Philippe Boutry, Jacqueline Carroy, Alain Corbin, Olivier Faure, JeanYves Mollier (président) et Michelle Perrot.

\section{Nicole Edelman}

Somnambules magnétiques et médiums ont constitué mon premier travail de recherche. C'était un champ encore peu travaillé par les historiens, s'il l'est en revanche beaucoup plus par les anthropologues et les sociologues : Françoise Champion, Danielle HervieuLéger, les anthropologues, Wiktor Stockowski, François Laplantine, Christine Bergé, Marie-Christine Pouchelle pour ne citer que ceux qui me sont les plus familiers. Il ouvre largement sur qu'on nomme l'occultisme et à ce titre demeure pour moi un espace de recherche à part entière. Voyantes, guérisseuses et visionnaires, mon premier livre, issu de ma thèse de doctorat, visait à comprendre le surgissement du magnétisme et du somnambulisme magnétique à la fin du XVIII ${ }^{\mathrm{e}}$ siècle et l'épanouissement d'une forme de clairvoyance et de transe au XIX ${ }^{\mathrm{e}}$ siècle. Je m'interrogeais à la fois sur la signification de ce sommeil qui plaçait au cœur de l'Occident chrétien une forme de transe originale et singulière et surtout sur le sens d'une telle posture pour une femme du XIX ${ }^{\mathrm{e}}$ siècle. Pour ce faire, je cherchais à saisir les conditions d'apparition et de transformation de ce savoir magnétique et somnambulique et à cerner pour les analyser les pratiques des groupes, cercles et autres sociétés que ce savoir créait, et je mettais au jour les enjeux sociaux, politiques, et scientifiques de tels mouvements.

Enjeu politique : le magnétisme somnambulique et le premier spiritisme ont bien des liens avec les socialismes de Fourier et Saint-Simon et ils sont sans doute combattu aussi à ce titre ; enjeu social : ainsi, le partage des rôles entre hommes et femmes est sous-jacent au discours et à l'activité des voyantes; enjeu scientifique enfin : l'opposition des conceptions magnétiques à celles de la médecine clinique "académique" est implacable. Et c'est bien pour cette raison que je passais de l'étude de la voyante à celle de l'hystérique, mon deuxième champ de recherche. Une grande partie des médecins était formelle : les 
voyantes sont des hystériques. Il s'agissait donc de comprendre pourquoi il en était ainsi et par quels cheminements on pouvait arriver à de telles conclusions. Là encore, si la médecine a de nombreux historiens, l'hystérie n'avait pas été interrogée à travers les métamorphoses de son étiologie et les enjeux dans lesquelles ces transformations étaient immergées.

Voyantes et hystériques, que j'ai prises successivement pour objets d'études sont deux figures dérangeantes et dissonantes dans le temps où elles vivent et à ce titre, elles révèlent l'état et l'évolution d'une société, ses modes de fonctionnements et ses dysfonctionnements. La voyante dérange par sa pratique, sa parole et ses écrits, par la place qu'elle tient à l'écart du privé. L'hystérique est gênante, elle interroge les règles et les interdits de la société ; sa maladie est problématique pour la médecine puisque sa symptomatologie ne cesse de changer dans un jeu d'inventions et de découvertes entre le médecin qui dit savoir et l'hystérique qui toujours déconcerte dans l'expression de sa demande et de sa souffrance.

Saisir le corpus. Comme la voyante, l'hystérique peut paraître insaisissable puisqu'elle n'est visible, perceptible qu'à travers ses représentations. En effet, l'histoire des sciences, même si le débat reste ouvert entre réalistes et constructionnistes, me semble clairement démontrer la fragilité de la vérité scientifique. Georges Canguilhem avait déjà avancé l'idée que l'histoire des sciences n'est pas l'histoire d'une progression vers la vérité, pas plus que l'histoire du vrai mais celle de discours véridiques. Il suffisait alors de retrouver les cheminements qui ont conduit à élaborer tel ou tel énoncé, en fonction d'une certaine norme interne à une activité scientifique donnée. En cela, l'histoire des sciences se constituerait quand même sur un autre mode que l'histoire en général. Et Michel Foucault souligne que c'est la "référence à l'ordre du vrai et du faux qui donne à l'histoire des sciences sa spécificité et son importance".

Pourtant dans l'histoire de l'hystérie et des métamorphoses de l'hystérique, cette référence au vrai et au faux est brouillée et fluctuante puisque, jusqu'à nos jours, le vrai n'a pas été découvert (même si la psychanalyse a profondément bouleversé la conception de l'hystérie). Il n'y avait donc pas d'appui possible sur une vérité scientifique. On sait que la Nature ne parle jamais d'elle-même, que ce sont toujours des hommes qui parlent en son nom, ce qui ne veut bien sûr pas dire que leurs énoncés en soient dissociés. Ainsi, à la suite de Dominique Pestre, je dirai que la science peut devenir "un dispositif qui produit et invente un ordre et non un dispositif qui dévoile l'ordre caché de la nature", le social se reconstruisant ainsi en permanence.

Les hystériques n'avaient donc d'existence, n'étaient visibles et perceptibles qu'à travers les réalités produites par d'autres, d'abord les médecins --et ils sont très prolixes-- mais aussi par tous ceux qui parlent de l'hystérie, les écrivains en particulier. Je pense en effet que ces récits fictifs nous renseignent sur le "réel" de l'hystérie même si ils n'ont que peu de rapport direct avec la réalité ; la fiction littéraire nous apporte alors "un inexprimable autrement". Le texte littéraire n'est ni témoignage, ni reflet, ni illustration, il est autre, et qui plus est, il est à son tour, capable de susciter du réel que les acteurs sociaux intériorisent et valident. Pour les hystériques, c'est patent. Le roman, le théatre, le GrandGuignol puisent ainsi leur inspiration dans les ouvrages théoriques des médecins, sur les amphithéâtres des hôpitaux et dans leurs salles communes, les chanteuses épileptiques copient les gestes et les attitudes des malades qui en retour amplifient et modifient les signes de leur pathologie. Flaubert comme Baudelaire, dans les années 1850, détournent le discours médical et dévoilent la difficulté de penser l'homme hystérique tout en l'inventant à l'encontre de la nosologie et de l'étiologie savante. Ils pensent, avant 
Charcot et différemment de lui, une virilité hystérique faite de passion et de transgression. Aux hommes la démesure hystérique féminine, celle de l'excès, du manque ou du débordement.

Pourtant, dans l'ensemble, le discours littéraire place et maintient l'hystérie du côté d'un détraquement du sexe féminin. La neurologisation radicale de l'hystérie, "névrose de l'encéphale", qu'effectue Pierre Briquet en 1859, n'est absolument pas entendue ni par les romanciers, ni par les poètes. En 1865, les Goncourt publient Germinie Lacerteux, invention romanesque d'une figure de l'hystérique plongée dans la trilogie du sang, des nerfs et du sexe. Zola place de même sa tante Dide, Adélaïde Fouque, grande ancêtre des RougonMacquart, de ce même côté. Le roman naturaliste s'appuie sur les théories médicales tout en les subvertissant, il représente une femme hystérique au corps détraqué, il témoigne de la sensibilité, de la nervosité de la femme et des blessures toujours possibles de son sexe. In fine, Phèdre et Salomé sont hystérisées. La littérature maintient l'hystérique, envers et contre toute nouvelle théorie médicale neurologique, comme Vénus tout entière à sa proie attachée.

Enfin l'Église s'intéresse à l'hystérie et des médecins catholiques théorisent à leur tour cette maladie surtout quand des médecins matérialistes expliquent dans la seconde moitié du XIX ${ }^{\mathrm{e}}$ siècle que possession diabolique et hystérie sont peut-être des symptômes équivalents. La pathologisation de la possession --dans certaines limites-- est acceptée et ce basculement débute à Morzine, lors des phénomènes qui touchent les femmes du village en 1857. En revanche, Église et médecins catholiques défendent l'extase et le miracle, essentiel à la croyance au surnaturel. La polémique prend des caractères aigus puis devient un combat quand les miraculées de Lourdes sont données par nombre de médecins positivistes, souvent grands patrons d'hôpitaux, Jean-Martin Charcot ou Hyppolite Bernheim, comme des hystériques guéries par suggestion.

Ce corpus lu et découvert, il fallait l'interpréter, mettre au jour l'interaction entre médecine et société, science et politique, entre normes sociales et normes scientifiques, entre les valeurs imposées et les discordances, comprendre le rapport entre ces normes et leur appréhension, leur intériorisation, restituer les tensions, les antagonismes. En allant le plus loin possible en amont des données pour saisir les constructions culturelles, médicales, sociales et politiques, percevoir enfin les rapports de dominations et l'ensemble des enjeux, cherchant non pas un contexte, ni même une contextualisation mais une historicité selon Michèle Riot-Sarcey.

Les métamorphoses de l'hystérique apparaissent ainsi comme révélatrices des peurs et de des questionnements du XIXe siècle mais aussi de ses évolutions culturelles, politiques et sociales, en particulier de la construction de la différence des sexes, de la recomposition permanentes des valeurs et des fonctions des hommes et des femmes, enfin de leur sexualité et finalement de leur existence en tant que sujet. Et plus simplement, dans le domaine de la médecine, elles permettent de découvrir les luttes entre aliénistes et neurologistes qui se déploient, lutte professionnelle, lutte pour le pouvoir au sein de l'institution hospitalière, soutenue ou non par le politique. Luttes qui induisent une vision de la maladie, la folie hystérique stigmatise combien plus que l'hystérie, maladie nerveuse.

L'hystérique, comme symptôme d'un état social, acquiert ainsi une grande visibilité à des moments particuliers de crises : dans les incertitudes et les doutes de la fin du XIX siècle, à Lourdes dans les années 1890, dans les salles des hystériques et épileptiques non aliénées du service de Charcot à La Salpêtrière dans les années 1870, à Morzine à la fin des années 1850. L'entremêlement entre social, religieux, médical et politique prend alors 
toute son ampleur. À Morzine, à la fin des années 1850, au moment où l'hystérique savante devient une malade respectable atteinte d'une maladie neuro-cérébrale, la figure de l'hystérique est marquée par le débordement et la transgression et le village se trouve confronté à la possession et au diable. Les médecins aliénistes sont alors envoyés, sommés de réagir sous la pression des pouvoirs tant médicaux que politiques à cette explosion de possession. Ils vont intégrer ces phénomènes à une nosologie hystérique et pathologiser pour la première fois la possession. L'hystérique devient alors une figure de référence, un cadre d'intelligibilité de ces déviances. Elle permet d'établir une norme scientifique qui, loin d'exclure ou de rejeter, assimile au contraire ces formes de révolte à une maladie bien définie et gomme ainsi leur caractère socialement dérangeant. En même temps, ces médecins aliénistes qualifient pour la première fois l'hystérique de folle cherchant à intégrer l'hystérie à leur savoir, ce qui ouvre le conflit entre généralistes, neurologistes et aliénistes. Par ailleurs, la question est aussi politique, les aliénistes dépendant en effet du ministère de l'Intérieur doivent ramener le calme et rétablir l'ordre public sans heurter la susceptibilité des notables savoyards qui viennent d'être rattachés à la France. Les Morzinoises troublent l'ordre public et mettent en cause l'ordre social dans une région rurale qui vient d'être annexée.

Enfin, l'avancée de la médecine dans l'espace religieux est une étape importante en ce qu'elle marque "le naufrage de la possession" qui était jusqu'alors une "catégorie recevable [qui] autoris[ait] l'expression du trouble psychologique et corporel". Seule la possession pouvait dire "le trouble dans le langage du mal et [donner] aux violences des possédées l'innocence de la souffrance". Le possédé était pris en charge par la communauté qui le "conduisait vers les instances propres à le délivrer". L'hystérique en revanche est face à elle-même, enfermée dans la violence corporelle de ses convulsions mais son délire ne peut plus référer à cet Autre diabolique. Ainsi "à partir de 1865, la symptomatologie, observée officiellement du mal de Morzine change de forme. La parole laisse place aux soubresauts et aux pantomimes muettes à l'Église. La douleur ne peut plus parler".

Pourtant la révolte ou le désarroi des Morzinoises s'étaient d'abord naturellement et culturellement coulés dans les formes d'expression connues de la possession. Sous couvert de possession, elles exprimaient sans doute leur révolte et leur mal-être en transgressant les règles élémentaires qui doivent régir la vie d'une femme et $a$ fortiori d'une jeune fille. La réponse que leur font les aliénistes est péremptoire et autoritaire. Leur comportement est lu comme un signe pathologique, elles sont définies comme hystériques ou folles hystériques, un diagnostic humiliant voire infamant. Ces femmes n'ont plus alors de communication possible avec leur communauté villageoise et semblent traduire cette impuissance dans les formes de leurs crises. Le diagnostic d'hystérie en dénouant les liens entre individues et communauté que créait la possession, dénie le sens des crises des Morzinoises dont les maux ne sont alors renvoyés qu'à elles-mêmes. L'Autre diabolique a disparu. Elles sont dites hystériques pour des raisons individuelles, privées voire intimes. Elles sont données comme folles de leur corps et dites folles tout court.

Avec évidence donc, les métamorphoses de l'hystérique ne s'appuient pas seulement sur le médical. Ainsi, après la Révolution française, Jean-Baptiste de Louyer-Villermay (1775-1837) définit l'hystérie comme une névrose génitale de la femme, liée à une lésion du système nerveux utérin, elle-même due à des besoins sexuels inassouvis. Mariage, et masturbation bien sûr péremptoirement condamnée, permettent la disparition de la maladie. Si Étienne Georget (1795-1828) réfère dès les années 1820 l'hystérie à une névrose cérébrale et si les médecins magnétiseurs interprètent eux aussi la maladie 
comme indépendante de la sphère génitale, ils ne sont pas entendus. La Restauration monarchique a poursuivi la re-construction d'une famille bien ancrée dans le catholicisme et ses valeurs et fondée sur la puissance du père, re-fondation, commencée sous le Consulat et validée par la promulgation du Code civil. Dans ce cadre, référer la femme à une nature excessive, ne met pas en danger le pivot social qu'est la famille, au contraire même, puisque l'hystérie cesse lors du mariage qui devient de facto une protection de la fragilité naturelle du Sexe.

Les choses changent singulièrement dans les années 1830, moment de tous les possibles, celui de la République entrevue par quelques-uns mais aussi temps des "réformateurs sociaux", tels les saint-simoniens ou les fouriéristes qui prônent une émancipation des femmes. D'autres théorisations de l'hystérie apparaissent alors; en 1830, celle de Frédéric Dubois d'Amiens (1799-1873) dont les travaux sont primés par l'Académie royale de médecine de Bordeaux. Dubois d'Amiens attribue lui aussi l'hystérie à une surexcitation nerveuse de la matrice qui n'est cependant plus liée à des passions sexuelles mais à l'exquise sensibilité des femmes, trop maternelles.

En 1845, c'est l'Académie royale de médecine qui donne un prix ex-aequo à Hector Landouzy et Jean-Louis Brachet (1789-1858), l'un et l'autre renvoient la maladie à des étiologies nerveuses ; Landouzy à une névrose de l'utérus qui induit des effets pathologiques sur le cerveau (et il n'exclut pas une hystérie masculine dont l'étiologie relève en ce cas d'une affection de l'appareil ganglionnaire génital mâle), Brachet à une névrose du système nerveux cérébral, l'hystérie masculine est, pour lui aussi, pensable mais elle ne concerne que des hommes efféminés dont le système nerveux est de sensibilité féminine. À cette date, l'hystérique devient donc une femme honorable, trop sensible, trop sentimentale, elle est moralement recevable. Le mariage n'est d'ailleurs ni pour Landouzy, ni pour Brachet une panacée. La femme doit simplement être protégée, surveillée par son mari ou son médecin. Fragiles, toutes les femmes sont, par nature, menacées d'hystérie. En ce domaine, le discours ne changera plus guère jusqu'au XX ${ }^{\mathrm{e}}$ siècle (et sans doute jusqu'au XXI ${ }^{\mathrm{e}}$ siècle).

Le libéralisme fait de la famille un pilier essentiel de la société et du politique, aussi bien sous la monarchie de Juillet que sous la $\mathrm{II}^{\mathrm{e}}$ République (sauf le court moment de février à juin 1848) et le Second Empire. Femmes et hommes sont pensés comme complémentaires, la mère-épouse représente le sentiment, elle est gage de moralité. Son excès sexuel se doit d'être gommé. La thèse neuro-génitale est ainsi largement défendue pendant les années 1850 par des "patrons" d'hôpitaux tels Négrier, Schutzenberger, Piorry qui pathologisent le plaisir sexuel des femmes en l'hystéricisant.

Les transformations de la figure de l'hystérique suivent en effet une stratégie de mise au silence ou du moins de mise en sourdine de toute expression du désir sexuel des femmes, les épouses et mères étant données comme modèles. À ce titre, P. Briquet en 1859 marque un point d'apogée à la fois de la neurologisation et de la désexualisation de la maladie puisqu'il est le seul à ne la référer ni à l'utérus, ni aux ovaires, il fait de l'hystérie une névrose de l'encéphale, il place donc l'étiologie de la maladie au niveau le plus haut du système nerveux. Il rapporte lui aussi la maladie à l'excessive sensibilité des femmes et construit une hystérique maternante, prolétaire et dolorosa.

Pourtant, dans ces mêmes années l'hystérique sort des hôpitaux, la plume des médecins n'en a plus l'exclusivité, écrivains et journalistes s'en emparent créant une parole bien souvent dissonante par rapport au discours médical et une parole fortement sexuée. Ce dualisme met d'ailleurs au jour les difficultés de la construction d'un modèle féminin dominant. Dans ce cadre, on comprend la difficulté de penser l'homme hystérique en tant 
qu'être viril, Georget, Brachet, Briquet le jugent concevable mais le définissent comme un efféminé, un inverti, pour Briquet même son existence est "le renversement des lois constitutives de la société". Or si Jean-Martin Charcot (1825-1893) élabore, pour la première fois, un hystérique viril et définit l'hystérie comme "une et indivisible", maladie hors du temps, partagée par les femmes et par les hommes, une maladie nerveuse comme les autres, les analyses des textes charcotiens infirment cette donnée : les hystériques femmes ne sont pas identiques aux hystériques hommes.

Pour comprendre ce paradoxe, j'ai posé la question en terme à la fois d'enjeux sociaux, au sens de la place des hommes et des femmes et d'une politique sociale et je me suis référée au modèle républicain, considérant que l'hystérie était une et indivisible, puisque Charcot l'affirmait, mais comme la République : les hommes et les femmes y étaient complémentaires et inégaux. Le modèle référentiel était cette fois la femme, l'Autre étant l'homme. Toutes les femmes étaient susceptibles d'être touchées par la maladie, en revanche, seule une catégorie d'hommes était menacée, les ouvriers, artisans, forgerons puis les vagabonds et les errants. Les hystériques masculins ne peuvent renvoyer qu'à une minorité pathologisée. Un homme --un vrai--, un citoyen, un électeur, un père ne peuvent pas être qualifiés d'hystériques, la femme --toutes les femmes-- peuvent l'être en revanche.

Quand Charcot meurt le 16 août 1893 dans une auberge du Morvan, l'hystérique neurocérébrale, l'hystérique à la fois homme et femme qu'il a voulu imposer, disparait vite car déjà vaincu-e par d'autres figures venues tant du monde médical que littéraire, qui n'ont jamais cessé de rôder tout autour. L'hystérie savante se délite, se défait à travers des étiologies et des nosologies qui se multiplient sans qu'aucun modèle ne s'impose, pas plus celui de Janet que de Babinski et son démembrement ou de Déjerine et moins encore, on le sait, celui de Freud. En revanche, la représentation publique de l'hystérique ne connaît plus guère de retouches. Hystérique est une injure adressée aux femmes car l'hystérique est indéniablement femme, qualificatif donné aux foules (féminines), l'hystérique se fige dans une figure de l'excès, à la nervosité extrême, frénétique, érotique et irresponsable. Ce passage de l'hystérie neuro-cérébrale à l'hystérie psychique correspond aussi à celui d'une interrogation sur la femme, sa place sociale, son rapport au masculin à une interrogation sur le sujet, un sujet cette fois universel et non plus seulement féminin. Ce basculement fin de siècle se situe au moment d'une crise qui se traduit par les doutes portés sur l'efficacité du libéralisme économique et politique, après le boulangisme, Panama et l'affaire Dreyfus, par la mise en cause de certitudes tant scientifiques que religieuses (le développement d'une nébuleuse de pensée de type occultiste en est une conséquence), enfin par la crainte d'une dégénérescence de la race française et d'un déclin démographique. Par ailleurs, la colonisation et la mainmise sur de nouveaux territoires invitent à re-penser l'être humain dans une diversité raciale et hiérarchisée qui justifie l'oppression des "colonisés", considérés comme des sous-hommes ou pas encore tout à fait des hommes.

À travers l'hypnose et la suggestion, Babinski, Bernheim, Janet et Freud, tous découvrent, à des degrés divers et avec des interprétations différentes, les feuilletages du moi de l'hystérique. Cette rupture médicale fait vaciller les convictions et les certitudes qui entourait l'unicité du Moi depuis les Lumières. Si la suggestion peut faire de chacun, hystérique ou pas, des sujets assujettis à un Autre, que faire du concept de l'individu libéral, doué de raison et "hors de l'état de tutelle" (Kant), condition de sa liberté ? Et le soupçon est aussi porté sur la conscience éclairée puisque l'individu peut se trouver déterminé à son insu, des affects refoulés ou enfouis pouvant resurgir sous des formes 
masquées dans la vie de chacun puisque dorénavant chacun possède quelque chose en lui qu'il ne maîtrise pas, un inconscient ou un subconscient.

Ainsi, les hystériques ne parlent plus seulement avec leurs corps qui cependant toujours se manifeste, signifiant physique essentiel de l'hystérie, mais elles-ils disent leurs souffrances et sont écouté-e-s. Dans le cadre de toutes les psychothérapies, celle de Déjerine comme celle de Janet ou celle de Freud (qui devient psycho-analyse), le médecin enjoint à l'hystérique de raconter son passé qui est alors écouté en tant que tel. Le malade devient un être qui parle. Janet reconstruit la vie de ses patient-e-s en y cherchant les idées fixes subconscientes isolées du champ de conscience et il préfère bien souvent les détruire par la suggestion sous hypnose ou les neutraliser en remplaçant une idée pathogène par une autre qui ne l'est plus sans toujours les mettre au jour de la conscience de son sujet. Déjerine recherche "l'action libératrice de la confession" puis vise à remodeler la vie de son patient, à "réorienter sa personnalité". Freud fait en sorte que le malade parvienne à vaincre la résistance qui s'oppose au retour de la représentation pathogène oubliées et maintenue hors de la conscience. Si en 1892, avec Breuer, dans les Études sur l'hystérie, il admet encore la suppression de cette représentation par suggestion médicale, en revanche, il ne cherche plus ensuite qu'à faire découvrir par le (Moi du) sujet lui-même le sens caché de ses symptômes, qu'à lui permettre de transformer sa "misère hystérique en malheur banal" pour, avec "un psychisme redevenu sain", être capable de mieux lutter contre ce malheur. Dans tous les cas, le médecin est indispensable pour décrypter et interpréter le signifiant du discours de l'hystérique dont il-elle doit encore reconnaître et accepter la vérité.

Du côté médical, le travail sur l'hystérique a ainsi permis l'émergence d'une nouvelle herméneutique, rupture décisive qui marque la naissance tout à la fois de la psychiatrie et de la psychanalyse. Les métamorphoses de l'hystérique ne s'arrêtent pas pour autant, si l'hystérique ne tient plus les premiers rôles, beaucoup se demandent qui l'a remplacée, l'anorexique ou les malades qui expriment ce syndrome de fatigue chronique pour lequel neurobiologiste et psychiatre s'opposent.

INDEX

Mots-clés : 2002

these 2002 